\title{
Consumo de bebidas alcoólicas e binge drinking nos jovens em formação
} Consumption of alcohol and binge drinking among school-age young invididuals Consumo de bebidas alcohólicas y binge drinking en jóvenes estudiantes

Olga Maria Martins de Sousa Valentim ${ }^{1}$ io https://oridi.org//0000-0002-2900-3972

Lídia Susana Mendes Moutinho² io https://orcid.org/0000-0001-5076-0612 José Carlos Marques de Carvalho ${ }^{3}$ io hitps://orcid.org/0000-0002-8391-8647

\section{Como citar:}

Valentim OM, Moutinho LS, Carvalho JC. Consumo de bebidas alcoólicas e binge drinking nos jovens em formacão. Acta Paul Enferm. 2021;34:eAPE01991.

DOI

http://dx.doi.org/10.37689/actaape/2021A001991

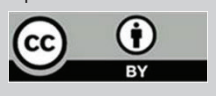

Descritores

Consumo de bebidas alcoólicas; Adolescente, Bebedeira

Keywords

Alcohol drinking; Adolescent; Binge drinking

\section{Descriptores}

Consumo de Bebidas Alcohólicas; Adolecente; Borrachera

Submetido 22 de Julho de 2019 Aceito 1 de Junho de 2020

Autor correspondente Olga Maria Martins de Sousa Valentim E-mail: ommvalentim3@gmail.com

\section{Resumo}

Objetivo: Este estudo, tem o objetivo de conhecer o consumo de bebidas alcoólicas e em particular a prática de binge drinking $(\mathrm{BD})$ em jovens que frequentam a escolaridade obrigatória, no distrito de Lisboa, Portugal.

Métodos: Estudo descritivo e correlacional de natureza quantitativa. Amostra constituída por 174 indivíduos de ambos os sexos que frequentam a escolaridade obrigatória em escolas do distrito de Lisboa, Portugal. Foram utilizados como instrumentos de avaliação: questionário sociodemográfico e Alcohol Use Disordens Identification Test (AUDIT-C). Análise dos dados pelo SPSS 25.

Resultados: Amostra com uma média de idades 15 anos, em que 75,9\% da amostra já consumiu bebidas alcoólicas e $23 \%$ da amostra já praticou BD, destacando-se uma maior percentagem no sexo feminino. Existem diferenças estatisticamente significativas entre a prática de $B D, 0$ consumo de risco identificado pelo AUDIT-C e 0 tipo de ensino frequentado. Participantes de ambos os sexos apresentavam percentagens idênticas de consumo de risco de bebidas alcoólicas. Há correlação positiva entre a idade de experimentação, o consumo de risco e a prática de $B D$. Conclusão: 0 consumo de bebidas alcoólicas nos jovens em formação, em idades muito precoces, assim como a de $B D$, mostram a pertinência de iniciar ações de prevenção do consumo de álcool ainda no ensino básico e a necessidade de aumentar a literacia dos jovens sobre o consumo de álcool, em especial no $B D$.

\section{Abstract}

Objectives: This objective of this study is to understand the consumption of alcohol, in particular the practice of binge drinking (BD), among young people attending compulsory education in the district of Lisbon, Portugal.

Methods: This was a descriptive correlational study of quantitative nature. The sample consisted of 174 male and female participants attending compulsory education in the district of Lisbon, Portugal. The following assessment instruments were used: a sociodemographic questionnaire and the Alcohol Use Disorders Identification Test (AUDIT-C). Data were analyzed using SPSS 25.

Results: The sample included participants with a mean age of 15 years old, $75.9 \%$ of which had already consumed alcohol, and $23 \%$ had already practiced BD; a higher percentage of BD was seen among female participants. Statistically significant differences correlating practice of $\mathrm{BD}$, hazardous drinking as per the AUDIT-C, and type of education attended were seen. Participants of either sex showed the same percentage of hazardous drinking. A positive correlation between age of onset, hazardous drinking, and BD was seen.

Conclusion: Early-onset alcohol consumption and BD among school-age young people evidences a pressing need for alcohol consumption prevention actions in Basic Education and increased alcohol consumption literacy among young people, in particular regarding BD. 


\section{Resumen}

Objetivo: Este estudio tiene el objetivo de conocer el consumo de bebidas alcohólicas, en particular la práctica de binge drinking (BD) en jóvenes que asisten a la escuela obligatoria en el distrito de Lisboa, Portugal.

Métodos: Estudio descriptivo, correlacional y de naturaleza cuantitativa. Muestra constituida por 174 individuos de ambos sexos que asisten a la escuela obligatoria en instituciones del distrito de Lisboa, Portugal. Los instrumentos de evaluación utilizados fueron: cuestionario sociodemográfico y Alcohol Use Disordens Identification Test (AUDIT-C). Análisis de datos mediante SPSS 25.

Resultados: Muestra con un promedio de edad de 15 años, de los que el 75,9 \% ya consumió bebidas alcohólicas y el 23 \% ya practicó BD, con un mayor porcentaje entre el sexo femenino. Existen diferencias estadísticamente significativas entre la práctica de $B D$, el consumo de riesgo identificado por el AUDIT-C y el nivel de enseñanza en el que se encuentran. Participantes de ambos sexos presentaron porcentajes idénticos de consumo de riesgo de bebidas alcohólicas. Hay una correlación positiva entre la edad que probaron por primera vez, el consumo de riesgo y la práctica de $B D$.

Conclusión: El consumo de bebidas alcohólicas de jóvenes estudiantes, a una edad muy precoz, así como la práctica de $B D$, demuestran la relevancia de iniciar acciones de prevención sobre el consumo de alcohol en la escuela primaria y la necesidad de aumentar la instrucción de los jóvenes sobre el consumo de alcohol, en especial el $B D$.

\section{Introdução}

O consumo de álcool em todo o mundo estima-se que afeta cerca de 2.3 bilióes de pessoas acima dos 15 anos. Estudos realizados em território nacional evidenciam descidas das prevalências do consumo de bebidas alcoólicas que se manifestam pela presença de indicadores positivos como o retardar das idades de início dos consumos nas populaçôes jovens, diminuição do consumo per capita e importantes ganhos em saúde ao nível da mortalidade e morbilidade. Contudo verificam-se percentagens preocupantes do binge drinking (BD), consumos de risco e dependência na faixa etária compreendida entre os 15 e os 34 anos. ${ }^{(1-3)}$

$\mathrm{Na}$ população mundial a prevalência de $\mathrm{BD}$ atinge o pico aos 20-24 anos à exceçấo da regiáo do Mediterrâneo Oriental onde as taxas de prevalência de BD entre bebedores de 15 a 24 anos são mais elevadas do que na população total. ${ }^{(1)}$

De acordo com o Inquérito nacional ao consumo de substâncias psicoativas na populaçáo geral (INPG), 2016/17 o BD, na faixa etária compreendida entre os 15 e os 24 anos é praticado por $11,8 \%$ da população. Ao comparar os dados apresentados pelo INPG nos anos 2012 e 2017 verifica-se uma redução de 5,75 pontos percentuais na faixa etária compreendida entre os 15 e os 24 anos no que se refere á prática de $\mathrm{BD}$. Este tipo de consumo é praticado maioritariamente pelo sexo masculino apesar de a diferença percentual entre sexos ser reduzida. ${ }^{(3)}$

O Estudo sobre o consumo de álcool, tabaco e drogas e outros comportamentos aditivos e dependências (ECTAD) realizado em 2015 na população escolar com idades compreendidas entre os 13 e os 18 anos mostra que a percentagem de consumidores de bebidas alcoólicas ao longo da vida vai aumentado proporcionalmente ao aumento da idade sendo que aos 18 anos $91 \%$ da populaçáo desta faixa etária já consumiu bebidas alcoólicas ao longo da vida e a diferença entre sexos é $0,6 \%$ superior no sexo masculino. ${ }^{(3)}$ A prática de $\mathrm{BD}$ também aumenta com o aumento da idade sendo que aos 18 anos e de acordo com a mesma fonte $36,2 \%$ da população tem este tipo de consumo nos últimos 30 dias, com maior número de praticantes no sexo masculino mas com uma diferença percentual de 12 pontos comparativamente ao sexo feminino. A comparação de $\mathrm{BD}$ entre os dados apresentados pelo ECTAD em 20111 e 2015 mostram um aumento deste tipo de consumo aos 17 e 18 anos o que contraria a tendência apresentada nas idades anteriores. $\mathrm{O}$ acentuar do $\mathrm{BD}$ aos 18 anos é também confirmado pelos resultados dos dados recolhidos no dia da Defesa Nacional a jovens de 18 anos, que mostra um amento do BD desde 2015 a 2017 nos últimos 12 meses. Salienta-se que de acordo com a mesma fonte, $49,5 \%$ dos jovens adotou este tipo de consumo nos últimos 12 meses. A prática de $\mathrm{BD}$ de acordo com os dados recolhidos em território nacional aumenta com idade sendo praticado nos últimos 12 meses por quase metade da população desta faixa etária. ${ }^{(3)}$

Nas últimas décadas, tem-se verificado uma modificação considerável no padrão de consumo de bebidas alcoólicas. Assim, passou-se do consumo diário de bebidas alcoólicas, essencialmente masculino e em quantidades frequentemente elevadas e por vezes com intoxicações agudas, como facilitador relacional 
e/ou integrado na dieta (consumo tendencialmente mediterrânico), para um consumo quase só nos finais de semana, muitas vezes com intenção de intoxicação aguda rápida (binge drinking), em idades cada vez mais precoces e cada vez mais no feminino (consumo mais próximo do anglo-saxónico). ${ }^{(4)}$

$\mathrm{O}$ uso de álcool caracteriza-se pela quantidade de álcool ingerido, período temporal em que a ingestão ocorre e consequências associadas ao consumo. Assim surgiu a necessidade de diferenciar um padrão típico de dependência de álcool de um comportamento de beber excessivo em uma única ocasião seguido por períodos de abstinência. $\mathrm{O}$ maior consenso foi alcançado sobre a definição do número de bebidas padrão e o período de tempo (número de horas e frequência) em que se verifica a ingestáo de álcool. ${ }^{(5)} \mathrm{O} \mathrm{BD}$ é usualmente definido como o consumo abusivo de cinco ou mais bebidas alcoólicas por homem, ou quatro por mulher, num período de duas horas. Em relação ao período temporal da avaliação do consumo, a maioria dos autores concorda que deve variar de 6 meses a 1 ano uma vez que este comportamento está parcialmente associado a eventos sociais, como festividades académicas, o que exige mais tempo para caracterizar adequadamente o padrão de ingestão de bebidas alcoólicas. ${ }^{(6,7)}$

Este tipo de consumo constitui um importante problema de saúde pública devido a complicaçóes decorrentes de comportamentos socialmente desajustados, tais como conduzir sob efeito de álcool, comportamentos sexuais de risco ou violência interpessoal, podendo também pode acarretar dificuldades de aprendizagem, insucesso escolar e absentismo no trabalho ou na escola. ${ }^{(8-10)}$

A idade em que se verifica a experimentação de bebidas alcoólicas assim como os primeiros episódios de $\mathrm{BD}$ corresponde à faixa etária em que em Portugal os jovens estão maioritariamente a realizar o seu percurso formativo. De acordo com a legislação portuguesa a frequência do ensino é obrigatória para as crianças e jovens com idades compreendidas entre os 6 e os 18 anos. Os jovens portugueses após concluírem o $9^{\circ}$ ano de escolaridade, mais ou menos aos 15 anos de idade dispóem de várias opções para continuar a sua formação. Estas passam pelo ingresso no ensino regular, tendo também como opção os
Cursos Profissionais, que se destinam aqueles que pretendem integrar o mercado de trabalho. Estes correspondem a $33,1 \%$ da população estudantil que frequenta a escolaridade obrigatória. ${ }^{(11,12)}$

Apesar de ser retratado em vários estudos um incremento acentuado do consumo de bebidas alcoólicas associados à integração no ensino superior simultaneamente constata-se que a idade de experimentação e as primeiras experiências de consumo do tipo $\mathrm{BD}$, acontecem durante a escolaridade obrigatória. Este estudo tem como objetivo conhecer o consumo de bebidas alcoólicas e em particular a prática de $\mathrm{BD}$ em jovens que frequentam a escolaridade obrigatória em escolas no distrito de Lisboa, Portugal. $(3,13,15)$

\section{Métodos}

O estudo é descritivo correlacional de natureza quantitativa. Os participantes foram selecionados em estabelecimentos do ensino secundário regular e profissional, utilizando a técnica de amostragem por conveniência. A amostra foi constituída por 174 participantes de ambos os sexos com idades compreendidas entre os 13 e os 22 anos de três escolas do distrito de Lisboa. Procedeu-se ao pedido de autorização aos diretores das diferentes instituições. Após a explicação do direito individual de recusar a participação a qualquer momento e sem consequências, e terem sido dadas garantias de confidencialidade dos dados recolhidos, bem como de anonimato dos respondentes, cada um dos participantes, pais e/ou responsáveis leu e assinou os Termos do Consentimento Informado antes da distribuição e preenchimento dos questionários. Foi obtida autorização da Comissão de Ética da Escola Superior de Saúde Atlântica para a realização do estudo e garantido o respeito por todos as recomendações éticas das declaraçóes de Helsínquia e Oviedo.

Os instrumentos de recolha de dados utilizados foram o Questionário Sociodemográfico que incluiu também as questóes "A que idade foi o primeiro consumo de bebidas alcoólicas?" e "Já alguma vez ingeriu bebidas alcoólicas?" e o The Alcohol Use Disorder Identification Test - C (AUDIT-C). ${ }^{(15,16)}$ 
The Alcohol Use Disorder Identification Test - C (AUDIT-C) é composto pelas três primeiras questôes do rastreio AUDIT,questionário aplicado internacionalmente e validado para a língua portuguesa, ${ }^{(17)}$ constituído por dez questóes que avaliam o consumo nocivo de álcool, sintomas de dependência e consequências nocivas da utilização abusiva de álcool. A pontuação AUDIT-C varia de 0 a 12 pontos, considerando-se o rastreio positivo. É recomendado a utilização dos seguintes pontos de corte para classificar um utente como tendo baixa probabilidade de consumo excessivo de álcool: sexo masculino com pontuação $<5$ pontos e sexo feminino pontuação $<4$ pontos. $^{(18)}$

A avaliação do consumo do tipo Binge drinking foi realizada através das respostas obtidas à pergunta 3 "Com que frequência consome seis bebidas ou mais numa única ocasião?” As respostas podem ser: 0 (nunca), 1 (menos de uma vez por mês), 2 (pelo menos uma vez por mês), 3 (pelo menos uma vez por semana) e 4 (diariamente ou quase diariamente). ${ }^{(18)}$

Para a comparação entre sexos e entre os dois tipos de ensino foi utilizado o teste $t(t)$ para amostras independentes. Para testar a correlação entre a idade do primeiro consumo e o risco de consumo de álcool e a prática de $\mathrm{BD}$, foi utilizado o Coeficiente de Correlação de Pearson (r). A análise dos dados foi realizada através do Statistical Packaje for the Social Sciences (SPSS), versão 25.0. Em todos os testes foi adotado o nível de significância de 5\% (p<0,05).

\section{Resultados}

A amostra foi constituída por 174 participantes, sendo $44,3 \%$ do sexo masculino e $55,7 \%$ do sexo feminino. A média de idades do sexo masculino é de $15,2( \pm 2,3)$ anos e no sexo feminino de 15,3 $( \pm 1,5)$. No que respeita ao tipo de ensino frequentado constatou-se que $75,3 \%$ frequenta o ensino regular e $24,7 \%$ o ensino profissional. Em relação ao consumo de álcool verificou-se que uma percentagem $75,9 \%$ da amostra já consumiu bebidas alcoólicas e apenas $24,1 \%$ diz nunca ter ingerido álcool. A idade do primeiro consumo oscilou entre os 5 e os 19 anos, sendo a idade com que mais frequência se verificou a experimentação foi aos 14 anos. A análise dos dados no que se refere á ingestão de bebidas alcoólicas no último ano mostra que 55,17\% dos participantes ingeriu bebidas alcoólicas (Figura 1).

No que diz respeito à identificação de participantes com baixa probabilidade de consumo excessivo de álcool, os resultados mostram que correspondem a $90,2 \%$ da amostra. O binge drinking

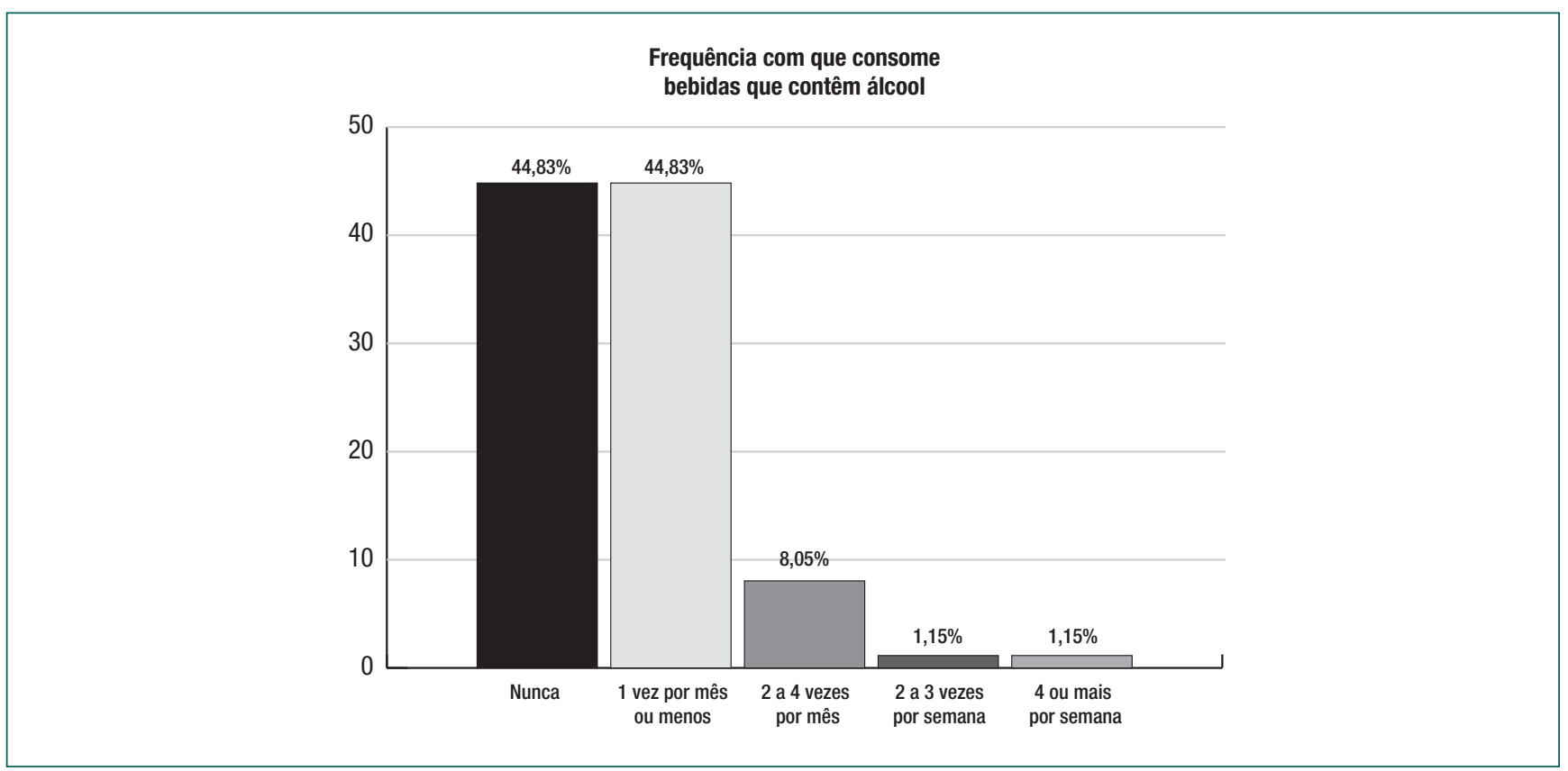

Figura 1. Frequência da ingestão de bebidas alcoólicas nos últimos 12 meses 
foi experienciado por $23 \%$ dos participantes, sendo que uma percentagem de $5,7 \%$ adota este padrão de consumo pelo menos uma vez por mês. Quando analisada a diferença entre sexos no que se refere há existência de consumos de risco de bebidas alcoólicas constatou-se que não há diferenças estatisticamente significativas ( $(14)=-0,60$ e $\mathrm{p}=0,55)$. As participantes do sexo feminino $(M=1,52 ; \pm 0,67)$ comparativamente ao sexo masculino $(M=1,33$; $\pm 0,76$ ), são os jovens que com mais frequência adotam o consumo de $\mathrm{BD}$, não havendo, no entanto, diferença estatisticamente significativa ( $\mathrm{t}$ $(37)=-0,82$ e $\mathrm{p}=0,41)$ entre sexos. Ao averiguar as diferenças entre os tipos de ensino frequentados pelos participantes constatamos que existem diferenças estatisticamente significativas ( $t$ (171) $=-3,47$ e $\mathrm{p}=0,00)$ no que se refere à idade do primeiro consumo, sendo que esta é mais precoce nos estudantes do ensino regular $(M=9,19 \pm 5,9)$ comparativamente aos que frequentam o ensino profissional $(M=12,7 \pm 5,6)$. A existência de consumos de risco de bebidas alcoólicas tem também significado estatístico ( $\mathrm{t}(15)=-2,15$ e $\mathrm{p}=0,05)$, sendo os alunos do ensino profissional os que apresentam scores de AUDIT-C mais elevados ( $M=6,42 \pm 2,07)$ comparativamente aos que frequentam o ensino regular $(\mathrm{M}=$ $4,70 \pm 1,25)$.Constatou-se que os participantes que tiveram uma experimentação, de bebidas alcoólicas, mais precoce, são os que apresentam uma prática mais frequente de $\mathrm{BD}(\mathrm{r}=0,25$ e $\mathrm{p}=0,001)$. A idade mais precoce de início dos consumos está também correlacionada positivamente com um score mais elevado do AUDIT-C ( $r=0,43$ e $\mathrm{p}=0,0001)$.

\section{Discussão}

O padráo de consumo de bebidas alcoólicas ao longo da vida $(75,9 \%)$ para a média de idades da amostra estudada situam-se entre os $31 \%$ e os $91 \%$ identificados por outros estudos realizados em território nacional. No entanto, a ingestão de bebidas alcoólicas no último ano $(55,17 \%)$ e a idade de experimentação mais tardia (Moda=14 anos) são superiores às identificadas, noutros estudos nacionais que apontam para os 13 anos..$^{(2,14,19)}$
A percentagem de BD (23\%) da amostra é superior, aos $15,4 \%$ apresentados por jovens de 15 anos em 2011 e aos 14,2\% em 2015. (3) o que parece contrariar a tendência para a estabilização dos consumos pelos jovens desde 2012. ${ }^{(1)}$

Os participantes com baixa probabilidade de consumo excessivo de álcool representam 90,2\% da amostra que é superior à encontrada noutros estudos realizados em Portugal. ${ }^{(2,19)}$ Nestes estão incluídos os que nunca consumiram bebidas alcoólicas (44,83\%) e os que consomem bebidas alcoólicas sem ainda apresentarem riscos para a saúde. É importante salientar que $55,17 \%$ dos participantes que ingeriram bebidas alcoólicas no último ano, 23\% adotou a prática de BD. Por certo, devido ao número de vezes que estes episódios ocorreram nos últimos doze meses, ainda não foi identificado pelo AUDIT-C como um consumo com riscos para a saúde.

A diferença percentual entre os participantes com consumos com probabilidade de riscos para a saúde $(9,8 \%)$ e os praticantes de $\mathrm{BD}(23 \%)$ parecem indicar a iliteracia dos jovens sobre as consequências do consumo de álcool independentemente do tipo de ingestão de bebidas alcoólicas. ${ }^{(20,21)}$

O tipo de ensino frequentado parece estar relacionado com a integração do consumo de álcool nos hábitos de vida dos jovens pois apesar de a idade de experimentação ser mais precoce nos estudantes do ensino regular comparativamente ao profissional, quando analisamos os consumos de risco verificamos que este valor é mais elevado nos que frequentam o ensino profissional. Por certo a perspetiva de integrar o mercado de trabalho com as responsabilidades inerentes e o assumir os papeis característicos da fase adulta (responsabilidades profissionais e gestão económica) poderão estar relacionadas com o tipo de consumo.

Os resultados mostram que a idade de experimentação apresenta correlaçóes estatisticamente significativas e positivas com a prática de $\mathrm{BD}$ $(\mathrm{r}=0,25$ e $\mathrm{p}=0,001)$ e com os valores mais elevados do AUDIT-C ( $r=0,43$ e $\mathrm{p}=0,0001)$. Estes dados parecem indicar que as medidas que visam atrasar a idade de experimentação devem ser associadas a outras estratégias que impeçam a integração do consumo de álcool nos hábitos de socialização dos jovens, 
pois apesar de iniciarem mais tarde o consumo, este é integrado sob a forma de $\mathrm{BD}$ ou com consumos com probabilidade de riscos para a saúde. ${ }^{(22)}$

\section{Conclusão}

O consumo de bebidas alcoólicas identificado na amostra em estudo, confirma a tendência que se verifica desde 2012 no atraso da idade de experimentação e percentagem de consumidores de risco, apesar de a percentagem de $\mathrm{BD}$ ser superior às apresentadas em outros estudos realizados em território nacional para a mesma faixa etária. $\mathrm{O}$ consumo de bebidas alcoólicas nos jovens em formação em idades precoces assim como o BD, mostram a pertinência de iniciar açôes de prevenção do consumo de álcool no ensino básico. Alertam também para a necessidade de aumentar a literacia dos jovens sobre o consumo de álcool e em especial no que se refere à prática de $\mathrm{BD}$. A realização deste estudo mostra que o consumo de álcool entre jovens durante a formação académica é influenciado por diferentes variáveis de que se destaca o tipo de ensino frequentado. É essencial o conhecimento dos fatores que influenciam o consumo de modo a serem planeadas e implementadas estratégias de prevenção adaptadas às necessidades apresentadas. Durante a formação educacional/académica dos jovens acontecem os primeiros consumos de álcool e em alguns casos a sua integração nos hábitos de vida. Nesta etapa de vida existem pessoas significativas tais como os professores ou líderes de atividades desportivas e recreativas que devem estar informadas sobre os sinais de consumo de substâncias e suas consequências de modo a ser possível a sua sinalização e encaminhamento dos jovens com consumos. É por isso relevante a realização de ações formativas direcionadas para os que lidam direta ou indiretamente com os jovens de modo a ser possível a identificação o mais precoce possível de consumos com riscos para a saúde. É fundamental que os profissionais da saúde, nomeadamente o enfermeiro, pesquisem sistematicamente e sem julgamentos prévios o uso do consumo de bebidas alcoólicas, para intervirem de forma individualizada e para diminuir o preconceito em relaçáo ao uso do álcool.

\section{Colaborações}

Valentim OMMS, Moutinho LSM e Carvalho JCM declaram que contribuíram com a concepção do projeto, análise e interpretação dos dados, redação do artigo, revisão crítica relevante de conteúdo intelectual e aprovação da versão final a ser publicada.

\section{Referências}

1. World Health Organization (WHO). Global status report on alcohol and health 2018. Luxembourg: WHO; 2018.

2. ESPAD Group. Report 2015. Results from the European school survey project on alcohol and other drugs. Luxembourg: Publications Office of the European Union; 2016.

3. Serviço de Intervenção nos Comportamentos Aditivos e nas Dependências (SICAD). Relatório Anual 2017. Situação do país em Matéria de Álcool. Serviço de Intervenção nos Comportamentos Aditivos e nas Dependências: Direção de Serviços de Monitorização e Informação / Divisão de Estatística e Investigação. Lisboa: SICAD; 2018

4. Portugual. Direção Geral da Saúde. Saúde mental em números 2013. Programa nacional para a saúde metal. Lisboa: Direcção Geral da Saúde; 2013.

5. Courtney KE, Polich J. Binge drinking in young adults: Data, definitions, and determinants. Psychol Bull. 2009;135(1):142-56.

6. Gunzerath L, Faden V, Zakhari S, Warren K. National Institute on Alcohol Abuse and Alcoholism report on moderate drinking. Alcohol Clin Exp Res. 2004;28(6):829-47.

7. Moutinho LS, de Oliveira Cruz Mendes AM, Lopes M. Alcohol consumption and binge drinking among young adults aged 20-30 years in Lisbon, Portugal. J Addict Nurs. 2018;29(4):E9-15.

8. Townshend JM, Kambouropoulos N, Griffin A, Hunt FJ, Milani RM. Binge drinking, reflection impulsivity, and unplanned sexual behavior: impaired decision-making in young social drinkers. Alcohol Clin Exp Res. 2014;38(4):1143-50.

9. Holway GV, Tillman KH, Brewster KL. Binge drinking in young adulthood: the influence of age at first intercourse and rate of sex partner accumulation. Arch Sex Behav. 2017;46(2):525-37.

10. Correas A, Cuesta P, López-Caneda E, Rodríguez Holguín S, GarcíaMoreno LM, Pineda-Pardo JA, et al. Functional and structural brain connectivity of young binge drinkers: a follow-up study. Sci Rep. 2016;6(1):31293.

11. Portugal. Ministério da Educação e Ciência. Decreto -Lei nº 176/2012. Diário da República n. ${ }^{0}$ 149/2012, Série I de 2012-08-02. 2012. Available from: https://data.dre.pt/eli/dec-lei/176/2012/08/02/p/dre/ $\mathrm{pt} / \mathrm{html}$

12. Engrácia P, Baptista JO. Situação após 3 anos dos alunos que ingressam no ensino profissional - anos letivos 2014/15, 2015/16 e 2016/17. D Lisboa: ireção-Geral de Estatísticas da Educação e Ciência (DGEEC); 2018. [citado 2020 Mar 10]. Disponível em: http://www.dgeec.mec.pt/ np4/\%7B\$clientServletPath\%7D/?newsld=952\&fileName=DGEEC_2018_ SituacaoApos3AnosEnsinoProfis.pdf 
13. Motos-Sellés P, Cortés-Tomás MT. Giménez-Costa Já. Evaluation of audit consumption items new adaptation to improve the screening of college students binge drinking. Adicciones. 2019;Mar 28:1145..

14. Sousa C, Almeida L. Consumo de álcool na transição do Ensino Secundário para o Ensino Superior. Atas do VI Seminário Internacional Cognição. Aprendizagem e Desempenho. Centro de Investigação em Educação, Universidade de Minho; 2018.

15. Inchley J, Currie D, Young T, Samdal 0, Torsheim T, Augustson L, et al. Growing up unequal: gender and socioeconomic differences in young people's health and well-being health behaviour in school-aged children (HBSC) study: international report from the 2013/2014 survey. Geneva: World Health Organization; 2016.

16. Anderson P, Gual A, Colom J. Alcohol and primary health care: clinical guidelines on identification and brief interventions. Barcelona: Department of Health of the Government of Catalonia; 2005.

17. Cunha J. Validação da versão portuguesa dos Questionários AUDIT e Five-Shot para identificação de consumo excessivo de álcool.
Faculdade de Medicina da Universidade de Lisboa. Lisboa: Internato Complementar de Clínica Geral da Zona Sul; 2002.

18. Direcção Geral da Saúde (DGS). Norma nº 030/2012. Deteção Precoce e Intervenção Breve no Consumo Excessivo de Álcool. Lisboa: DGS; 2012

19. Gual A, Segura L, Contel M, Heather N, Colom J. Audit-3 and audit-4: effectiveness of two short forms of the alcohol use disorders identification test. Alcohol Alcohol. 2002;37(6):591-6.

20. Neto C, Fraga S, Ramos E. Consumo de substâncias ilícitas por adolescentes portugueses. Rev Saude Publica. 2012;46(5):808-15.

21. Reis A, Barros J, Fonseca C, Parreira L, Gomes M, Figueiredo I, et al. Prevalência da ingestão de álcool nos adolescentes: estudo PINGA. Rev Port Clin Geral. 2011;27(4):338-46.

22. Loureiro LM, Barroso TM, Mendes AM, Rodrigues MA, Oliveira RA, Oliveira NR, et al. Literacia em saúde mental de adolescentes e jovens portugueses sobre abuso de álcool. Esc Anna Nery. 2013;17(3):47481. 